\title{
Cardiopulmonary Signal Detection Based on Magnetic Induction
}

\author{
Dan Yang, ${ }^{1}$ Bin Xu, ${ }^{2}$ Hongyu Qiao, ${ }^{1}$ and Xu Wang' \\ ${ }^{1}$ College of Information Science and Engineering, Northeastern University, Shenyang 110819, China \\ ${ }^{2}$ College of Computer Science and Information Engineering, Northeastern University, Shenyang 110819, China
}

Correspondence should be addressed to Dan Yang; yangdan@mail.neu.edu.cn

Received 6 December 2016; Revised 14 April 2017; Accepted 16 May 2017; Published 14 June 2017

Academic Editor: Giorgio Pennazza

Copyright (C) 2017 Dan Yang et al. This is an open access article distributed under the Creative Commons Attribution License, which permits unrestricted use, distribution, and reproduction in any medium, provided the original work is properly cited.

\begin{abstract}
Cardiopulmonary signal contains vital and rich physiological information, which is very useful for clinical diagnosis and home healthcare. Convectional monitoring methods still need uncomfortable sensors or are expensive for nonprofessional common consumers. In this paper, we proposed a cardiopulmonary signal detection method based on electromagnetic induction. Based on thoracic volume variation affecting the biological impedance, which can be detected by magnetic induction, our idea is to develop a not complicated and practical measuring platform and exploit the intrinsic properties of cardiopulmonary signals with respiratory and heart rate. We have some theoretical analysis about the relationship of thoracic volume with biological impedance, sensor-head parameters, and the optimal measuring position. Then a whole measuring system has been designed and evaluated. The respiratory and heart rate obtained from the proposed method are not significantly different from the reference method (noncontact BCG).
\end{abstract}

\section{Introduction}

According to the World Health Organization (WHO), cardiovascular diseases (CVD) are the leading causes of death and disability in the world. An estimated 17.5 million people died from CVD in 2016, representing 30\% of all global deaths [1]. Meanwhile the elderly population has increased to almost 810 million in 2012. In 2050, the number of aged people (60 and above) is about to reach a staggering 2 billion [2]. These cause the needs to monitor patient health status while they are out of the hospital to increase the comfort and wellbeing of the patients which can be in a familiar environment and at the same time decrease the significant cost on healthcare. Effective disease management through continuous monitoring and information fusion of vital physiological signals in patients is viewed as the key mechanism for the mentioned problems.

Cardiopulmonary signal includes respiratory and heart rate information that can be monitored to detect acute changes in personal condition (e.g., apnea) and potentially provide an early warning of impending life-threatening deterioration. Current methods require sensors attached to the body or not robust to subject motion. The most widely used method for detecting cardiopulmonary signal is electrocardiography (ECG) which provides useful information about the cardiovascular system. ECG captures the electrical activity of the heart as a time series data with $\mathrm{Ag} / \mathrm{AgCl}$ electrodes attached to exposed bare skin; this will cause restraint and produce some burdens to subjects [3]. Airflow is another popular measuring vital sign (respiratory) method with common sensors fixed at the nasal cavity or the chest [4]. Video camera methods run the gamut ranging from high-end expensive camera systems that track bright objects placed on the chest and used in CT image gating to low-cost vital signs "apps" for mobile devices that give heart rate by detecting facial flushing with each beat and respiration rate by chest movement [5]. Thermal cameras detect air movement and temperature effects, while radar and Wi-Fi disturbance methods detect chest wall motion [6, 7]. UWB Doppler radar monitors vital signs of a person in a noncontact manner by coherently processing the time history of narrow pulses emitted by the radar and subsequently backscattered by the human chest [8]. These sensor-attached methods are uncomfortable and inconvenient for mobile applications. They 
cannot be also applied to all patients. In addition, contactless devices monitoring the cardiopulmonary signal are more professional, which are not affordable for home-based use. Therefore, in this paper we prefer to detect cardiopulmonary signals without contact, especially for home, nonburden, ambient-assisted living and long-term monitoring.

Magnetic induction is a valuable measurement contactless modality based on eddy current induction, which has been used to monitor human physiological information during the last decade. An alternating magnetic field is generated by excitation coils, which induces eddy currents into the conductive tissue of a body. Usually the diseased tissue of the organism will produce differences in conductivity, and this change could be reflected in the characteristics of the induced magnetic field, including frequency, amplitude, and phase. Then the pathological and physiological condition of biological tissue was analyzed by detecting the induced magnetic field. Previously, several approaches have been proposed to monitor human physiological signals with magnetic induction techniques. In [9], the authors proposed a simple measurement hardware holding with actual excitation and measurement coils. Testing experiments have been on a healthy adult volunteer who was resting on bed. However, the testbed is not equipped in the normal bed, and some manual postprocessing is required. In [10-12], they present a simulation model with a six-channel axial coil gradiometer underneath a neonatal thorax. They analyze the coverage of the whole bed, induction into the body, and distance between measuring points. Some animal trials have been done for evaluating the feasibility of monitoring vital signs by magnetic induction. They just discussed the feasibility by more simulating. Alternatively. In $[13,14]$, reported the related experiment of using magnetic induction methods to place sensors under the bed for physiological signal detection. The measuring principles are based on phase difference between exciting coil and receiving coil. Teichmann et al. in $[15,16]$ discussed the capacitive or inductive coupling for recording cardiorespiratory activity. The proposed system contains inductive sensor head and capacitive sensor head. The experiments reveal the inductive sensor is sensitive to tissue conductivity.

Inspired by these prior works, we aim to improve and develop a home-used, feasible cardiorespiratory monitoring system based on magnetic induction. We consider the thoracic volume can be varied by physiological activity especially due to heart or lung breathing motion, and this will change the conductivity with the thoracic volume. In our solution, the only sensor-head coil is used to couple biological impedance change. The human body is connected in the oscillation tank circuit by coil inductive sensing thoracic volume variation. The oscillation frequency will change with thoracic equivalent impedance change caused by cardiopulmonary activity. The contribution of our work is as the following: we proposed a noncontact cardiorespiratory signal monitoring technique based magnetic induction, which exploits the intrinsic properties of detecting signal about respiratory or heart rate. And we enable the only sensor head in our designed system to obtain the useful information and simplify the conventional system hardware design. The

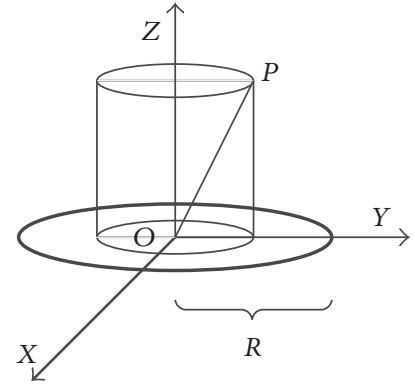

FIgURE 1: Single coil detection system coordinate.

proposed method is thoroughly evaluated by the theoretical analysis and the laboratory tests and also demonstrates comparable performance against guided breath behavior or the BCG method.

\section{Magnetic Induction Measuring Model}

2.1. Materials' Conductivity Variation $\Delta \sigma$ Related to the Impedance Variation $\Delta Z$. The $z$-component of magnetic field $B$ from a coil of $N$ turns, carrying AC current $I e^{j \omega t}$ centered on the $x$-y-axis, as seen in Figure 1 , can be described by the Biot-Savart law:

$$
\begin{aligned}
& B_{z}(\rho, z, t) \\
& \quad=\frac{\mu_{0} N I e^{j \omega t}}{4 \pi} \int_{0}^{2 \pi} \frac{(\rho-\rho \cos \theta)}{\left(\rho^{2}+R^{2}+z^{2}-2 \rho R \cos \theta\right)^{3 / 2}} d \theta,
\end{aligned}
$$

where $\mu_{0}=4 \pi \times 10^{-7} \mathrm{~N} / \mathrm{A}^{2}$ is the permeability of free space and $\rho$ is the radius from the $z$-axis $j=\sqrt{-1}, \rho=\sqrt{x^{2}+y^{2}}$, $\theta=\tan ^{-1}(y / x)$

The $z$-component of the induced magnetic field by the single turn coil can be denoted as

$$
B_{s z}=C_{1} \int_{L_{1}}^{L_{2}} d z \int_{0}^{r(z)} d \rho \int_{0}^{\rho} d \rho^{\prime} \int_{0}^{\pi} d \theta \int_{0}^{\pi} d \alpha\left[F_{1}\right] \omega,
$$

where

$$
\begin{aligned}
& \Delta Z=32 \pi^{3} \times 10^{-14} N^{2} f^{2} R^{3} \widehat{I}[\Delta \sigma+j \omega \varepsilon] \\
& =32 \pi^{3} \times 10^{-14} N^{2} f^{2} R^{3} \widehat{I} \Delta \sigma+j 64 \pi^{3} \times 10^{-14} N^{2} f^{2} R^{3} \widehat{I} \omega \varepsilon, \\
& \Delta Z=32 \pi^{3} \times 10^{-14} N^{2} f^{2} R^{3} \widehat{I}[\Delta \sigma+j \omega \varepsilon] \\
& =32 \pi^{3} \times 10^{-14} N^{2} f^{2} R^{3} \widehat{I} \Delta \sigma+j 64 \pi^{3} \times 10^{-14} N^{2} f^{2} R^{3} \widehat{I} \omega \varepsilon \\
& =\Delta f+j \Delta X, \\
& F_{1} \\
& =\frac{\rho^{\prime}\left(R^{2}-\rho^{\prime} R \cos \theta\right) \cdot(\rho-h \cos \alpha)}{\left(R^{2}+\rho^{\prime 2}+z^{2}-2 \rho^{\prime} R \cos \theta\right)^{3 / 2} \cdot\left(h^{2}+\rho^{2}+z^{2}-2 h \rho \cos \alpha\right)^{3 / 2}} .
\end{aligned}
$$

The magnetic flux $\Phi_{s}$ of the circle area with the radius $R$ and symmetry $z$-axis is

$$
\Phi_{s}=\int_{0}^{R} B_{s z}\left(2 \pi \rho^{\prime}\right) d \rho^{\prime} .
$$




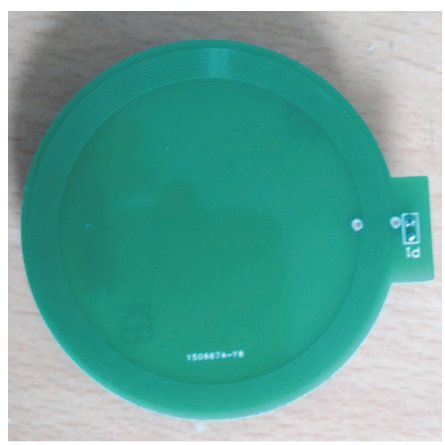

(a) The designed circular plane coil sensor

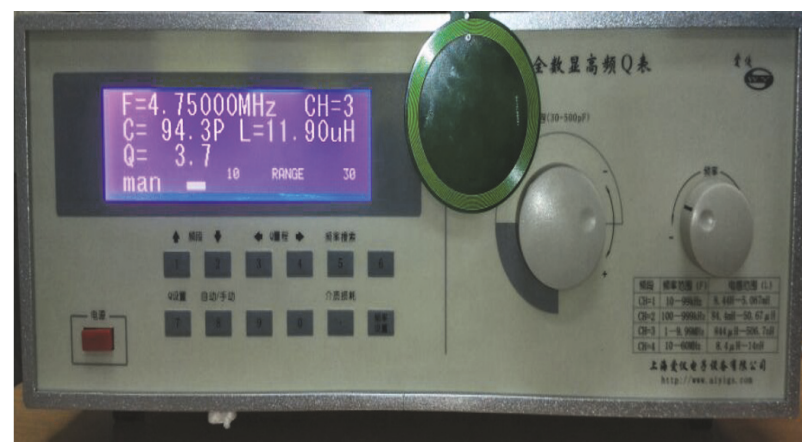

(b) The inductive value of designed coil by $Q$ meter high frequency

FIgURE 2: The coil sensor head and measuring device.

The variation of induced electromotive force (EMF) in the coil sensor is

$$
\Delta E=-N \frac{d \Phi_{s}}{d t}=-j \omega N \Phi_{s} .
$$

The induced EMF also can be denoted by

$$
\Delta E=-\left(I e^{j \omega t}\right) \Delta Z \text {. }
$$

Then

$$
\Delta Z=-\frac{\Delta E}{I e^{j \omega t}}=C_{2} \widehat{I}
$$

where

$$
\begin{aligned}
& C_{2}=32 \pi^{3} \times 10^{-14} N^{2} f^{2} R^{3}[\sigma+j \omega \varepsilon), \\
& \widehat{I}=\int_{L_{1} / R}^{L_{2} / R} d z \int_{0}^{r(z)} d \rho \int_{0}^{\rho} d \rho^{\prime} \int_{0}^{1} d h \int_{0}^{\pi} d \theta \int_{0}^{\pi} d \alpha, \\
& F \\
& =\frac{\rho^{\prime}\left(1-\rho^{\prime} \cos \theta\right) \cdot h(\rho-h \cos \alpha)}{\left(1+\rho^{\prime 2}+z^{2}-2 \rho^{\prime} \cos \theta\right)^{3 / 2} \cdot\left(h^{2}+\rho^{2}+z^{2}-2 h \rho \cos \alpha\right)^{3 / 2}} .
\end{aligned}
$$

The relationship between the coil impedance with the material conductivity and dielectric constant is

$$
\begin{aligned}
\Delta Z= & 32 \pi^{3} \times 10^{-14} N^{2} f^{2} R^{3} \widehat{I}[\Delta \sigma+j \omega \varepsilon] \\
= & 32 \pi^{3} \times 10^{-14} N^{2} f^{2} R^{3} \widehat{I} \Delta \sigma+j 64 \pi^{3} \\
& \times 10^{-14} N^{2} f^{2} R^{3} \widehat{I} \omega \varepsilon=\Delta R+j \Delta X,
\end{aligned}
$$

where $\omega$ is current frequency and $\varepsilon$ and $\sigma$ are the material relative conductivity and dielectric parameter. For biological tissues, the real part of $\Delta Z$ is far greater than the imaginary part of $\Delta Z$; then

$$
\Delta Z \approx \Delta R, \quad \Delta Z \propto \Delta \sigma
$$

\section{Hardware Platform}

In our design, the hardware platform consists of coil sensor head, signal receiver unit, and signal controlling unit. The coil

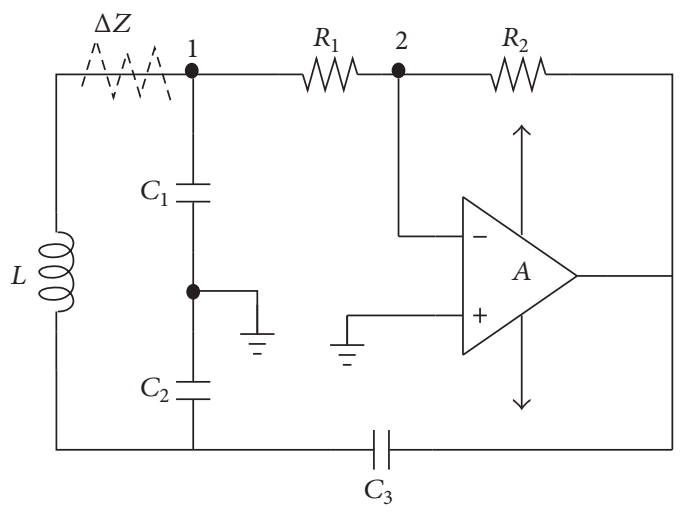

FIGURE 3: Colpitts oscillating circuit considering inductive coil $L$ and varying body impedance $\Delta Z$.

sensor works for coupling the impedance change. The signal receiver unit includes LC oscillator circuit and postprocessing circuit, which respond for sampling the oscillating frequency related to the target conductivity variation. The signal controlling unit is built with the central control processor and display part used for controlling signal receivers, displaying the results and communicating with PC for all further signal processing steps.

3.1. Coil Sensor Head. In our system, we design the circular plane PCB coil for coupling the body conductivity change. The coil turn is 10 , the average radius is $2.9 \mathrm{~cm}$, and the width and distance of wire line both are $0.0254 \mathrm{~cm}$, shown in Figure 2(a). The theoretical inductance is $12.08 \mu \mathrm{H}$. The practical inductance value of our coil is $11.90 \mu \mathrm{H}$ by high frequency $Q$ meter (shown in Figure 2(b)).

3.2. Signal Receiver Unit. In our solution, the detecting signal unit works by detecting the change in coil sensor impedance caused by motion in the heart and lung. The oscillator frequency variation is related to the body conductivity change. The coil sensor is connected in Colpitts oscillating circuit. The schematic diagram is shown in Figure 3, where $L$ denotes the 
TABLE 1: Human thorax volume at various cardiopulmonary activity for male and female.

\begin{tabular}{lcccc}
\hline & Inspiration & Normal breath & Expiration & Holding breath \\
\hline Volume change/L & 1.25 & 0.25 & 0.75 & \\
Equivalent radius/cm & & & & 0.75 \\
Male & 8.85 & 7.33 & 5.37 & 6.94 \\
Female & 8.64 & 7.03 & 4.74 & 6.6 \\
\hline
\end{tabular}

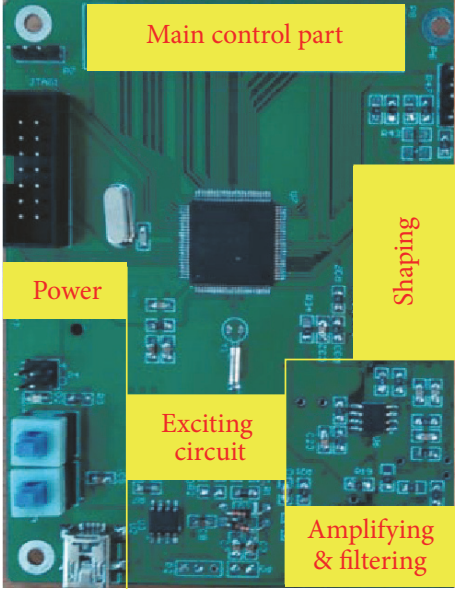

(a)

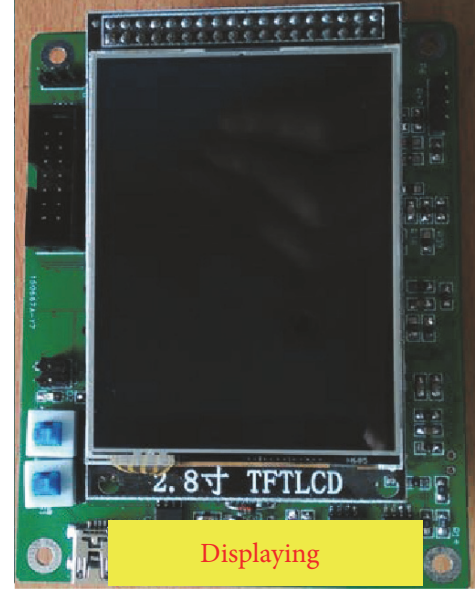

(b)

FIgURE 4: Hardware platform: (a) main board and (b) TFTLCD.

coil sensor inductance, $\Delta Z$ denotes the human body conductivity change, and $R_{1}, R_{2}, R_{3}, C_{1}$, and $C_{2}$ are the circuit parameters. The oscillator frequency $\omega_{\Delta Z}$ can be denoted by the equation

$$
\begin{aligned}
& \omega_{\Delta Z} \\
& =\sqrt{\frac{C_{1}+C_{2}\left(\left(R_{1}+\Delta Z\right) / R_{1}\right)+C_{3}\left(\left(R_{1}+R_{2}+\Delta Z\right) / R_{1}\right)}{C_{1} L\left(C_{2}+C_{3}\right)}} .
\end{aligned}
$$

When the circuit is powered, the resonance frequency is $\omega_{0}$ just caused by the coil sensor impedance $L$. Then the whole circuit is placed near the target object. The thoracic variation of target object due to heart and lungs motion will cause the body conductivity change; correspondingly the equivalent impedance of human body will be varied $\Delta Z$. Then the oscillator frequency of the Colpitts oscillator circuit will be $\omega_{0}+\omega_{\Delta Z}$. For the value of varied impedance, $\Delta Z$ is very small and the signal of the frequency change $\omega_{\Delta z}$ will be very weak. In order to detect the faint frequency change, the chip OPA690 was selected to be configured as postprocessing circuit for amplifying and filtering. OPA690 is unity-gain stable, voltage-feedback op amps. The amplifier circuit gain is 10, and the output is connected to the other OPA690, designed as Butterworth active filter with cutoff frequency 5.1 Mhz. For counting the frequency, the waveform transfer circuit is designed for shaping the frequency waveform.

3.3. Signal Controlling Unit. The signal controlling unit includes primary control processor and LCD. The main control board using chip MSP430F5438A is responsible for data receiving and communication with $\mathrm{PC}$. The control chip clock frequency is set as $24 \mathrm{MHz}$. Thin Film Transistor (TFT) is used for displaying the detecting frequency signal waveform. The whole board has a dimension of $9.2 \mathrm{~cm} \times$ $7.4 \mathrm{~cm}$. The power supply is $3.3 \mathrm{~V}$ obtained by AMS1117. The whole designed system is shown in Figure 4.

\section{Experiments and Results}

4.1. Cardiopulmonary Activity and Impedance Changes. According the physiological data, human thorax is supposed as the sphere. Table 1 lists the human thorax volume at various cardiopulmonary activity for male and female. During the simulation study, the distance between the coil and the thorax sphere is $10 \mathrm{~cm}$, coil turn is 1 , and the radius of coil is $6 \mathrm{~cm}$. The oscillation frequency is $5 \mathrm{Mhz}$. The relationship between the changes of thoracic volume and the detection impedance was simulated, shown in Figure 5. During the process of deep inspiration exercise, the change of impedance was increased obviously. The main reason was that cardiopulmonary activity was obvious and the change of electrical conductivity was obvious in deep breathing process. And compared to women, men change a little more, which with their own physiological reasons have a certain relationship.

4.2. Coil Parameters. In order to measure the cardiopulmonary signal efficiently, the coil sensor parameters are very important such as the radius and measuring position. The 


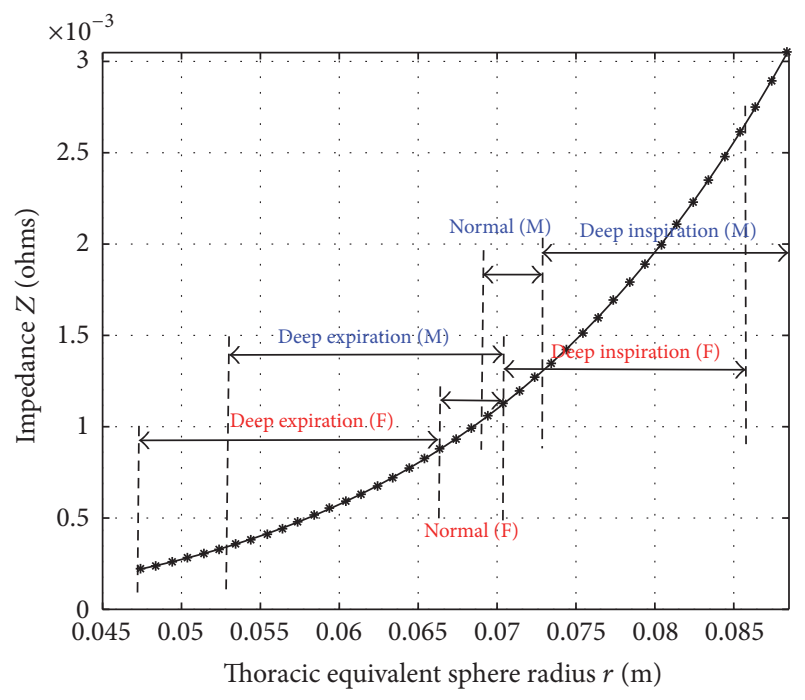

Figure 5: The effect of the thoracic radius and impedance.

saline was used for analyzing the coupling degree between the coil and the object signal. We simulated the testbed for discussing the coil sensor parameters. In the simulated testbed, coil sensor was placed below the cylindrical cylinder, which is used to contain $1 \mathrm{~cm} / \mathrm{ms}$ saline. There was a small vessel, which is suspended in the middle of the cylindrical cylinder by manual controlling. The cylinder diameter and sample vessel diameter were $5 \mathrm{~cm}$ and $4 \mathrm{~cm}$, respectively. The conductivity range in small vessel representing different types of tissue and organs $\sigma_{s}=1 \sim 8 \mathrm{~cm} / \mathrm{ms}$.

Figure 6(a) shows the relationship between the signal output $\Delta f$ of the coil sensor by the object saline $\sigma_{s}=8 \mathrm{~cm} / \mathrm{ms}$ at the distance $h$. The results are based on different oscillation frequency and coil turns. At the same measuring condition, the higher the oscillation frequency and the greater the number of coil turns, the larger the couple frequency change output. There is a small oscillation in the tail of the curve. This may be caused by the sample vessel moving or manual operation factors (such as moving speed and hand shaking). Figure 6(b) shows the influence of coil sensor radius change for the couple frequency. The greater the coil sensor radius, the higher the simulated system's sensitivity. But when the distance between the coil and target object is near $2 \mathrm{~cm}$, for the target small vessel radius is $2 \mathrm{~cm}$, the radius $r=1.5 \mathrm{~cm}$ coil sensor has better sensitivity.

In order to improve the system sensitivity, the optimal position of coil sensor has been discussed. The object body tank is represented by the cylinder $\sigma_{\text {tunk }}=0.3 \mathrm{~s} / \mathrm{m}$, the heart is simulated by the sphere $\sigma_{\text {heart }}=0.57 \mathrm{~s} / \mathrm{m}$, and the trapezoid cylinder represented the lungs $\sigma_{\text {lung }}=0.35 \mathrm{~s} / \mathrm{m}$. There are six positions to be considered, shown in Figure 7(a). The sphere with radius $40 \mathrm{~cm}$ is the border, the cylinder represents the human body, the two cylindrical cylinders are denoted as lungs, and the small sphere is simulated as a heart. Positions 1 and 2 located in the lungs, position 3 located in the middle of heart, position 4 located in the boundary between the lung and heart, position 5 located in the middle of left lung and right lung, and position 6 is far from the lung, shown as

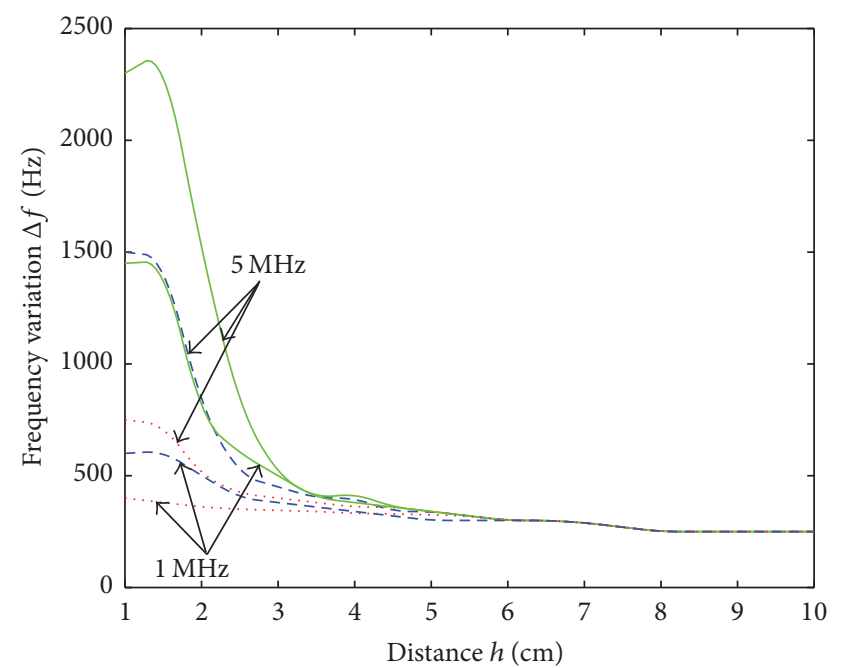

$$
\begin{aligned}
& n=10 \text { at } 5 \mathrm{MHz} \quad n=10 \text { at } 1 \mathrm{MHz} \\
& \ldots n=5 \text { at } 5 \mathrm{MHz} \quad \ldots n=5 \text { at } 1 \mathrm{MHz} \\
& n=3 \text { at } 5 \mathrm{MHz} \quad \ldots \ldots n=3 \text { at } 1 \mathrm{MHz}
\end{aligned}
$$

(a) The sample conductivity is fixed; the fundamental frequency and the number of coil turns are varied

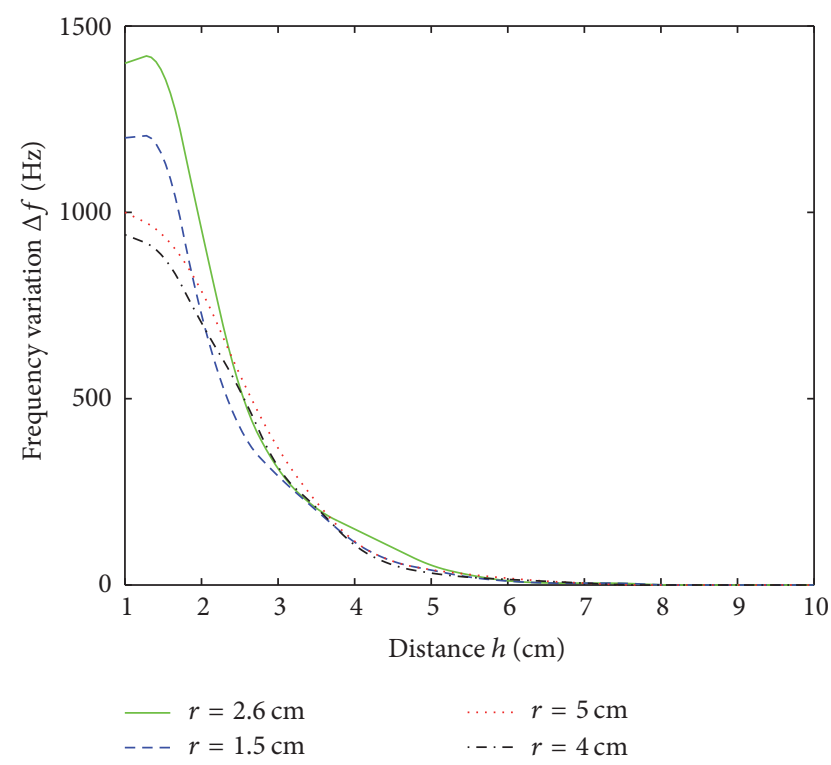

(b) Only change in the coil radius

FIGURE 6: The relation of signal output frequency with coil to object distance $h$.

Figure 7(a). Setting the heart as the center, we set 5 points at both sides in horizontal and vertical distribution. Each point is spacing $2 \mathrm{~cm}$. Figure 7(b) shows the imaginary part of magnetic flux density when the coil sensors at different six positions move along the horizontal axis. The coil sensor is placed at various positions, and the variation in the value of the magnetic flux density is very small. The change is much higher when the coil sensor located at various horizontal location. At the distance to the center $6 \mathrm{~cm}$, the magnetic flux density is largest. The value of position 3 is greatest due to heart conductivity, the measuring range of coil sensor 

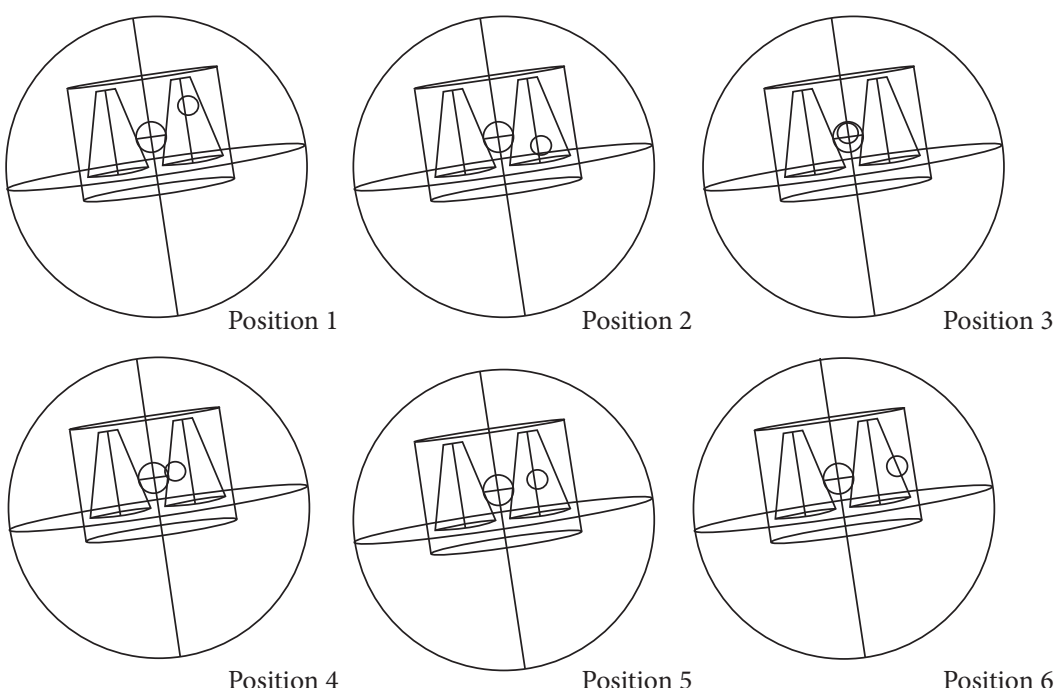

(a)

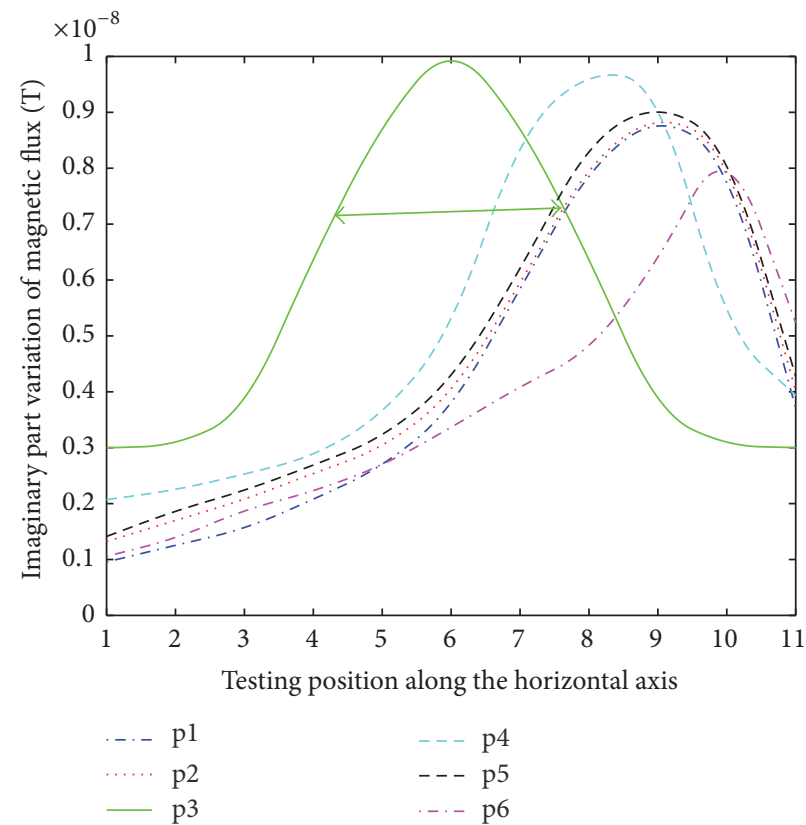

(b)

Figure 7: Simulated coil sensor position at COMSOL and MATLAB. (a) Simulated coil sensor position at COMSOL. (b) Comparison of different coil positions in MATLAB.

moving is greater, and the signal contains much integrity. Then position 3 is selected as best position.

\subsection{System Evaluation}

4.3.1. Frequency Change Detecting. The proposed cardiopulmonary signal measuring board can be fixed on target object clothes, or sitting chair, or lying bed. The measured position should be near the human body, especially not too far as the discussed position 3. In our experiment, we evaluate the designed system at sitting state by the testbed. A young normal girl was asked to wear the test board on the backside (sternal left $2 \mathrm{~cm}$ ), shown as Figure 8(a). Regular daily activities were performed: standing and sitting. Figure 7 shows the result of one experiment in which the human subject follows instructions to perform various cardiorespiratory activities. In TFT displaying the $x$-axis is representing the time unit second, and the $y$-axis described the frequency unit $\mathrm{MHz}$. At first, the subject was asked to perform normal breath $12.5 \mathrm{~s}$ and then deep inspiration $7.5 \mathrm{~s}$ when she was sitting on the chair. It clearly shows that the frequency change in normal breathing is about $200 \mathrm{~Hz}$ and in deep inspiration is $280 \mathrm{~Hz} 360 \mathrm{~Hz}$, shown in Figure 8(b). We also observed the cardiorespiratory variation when subject was standing 


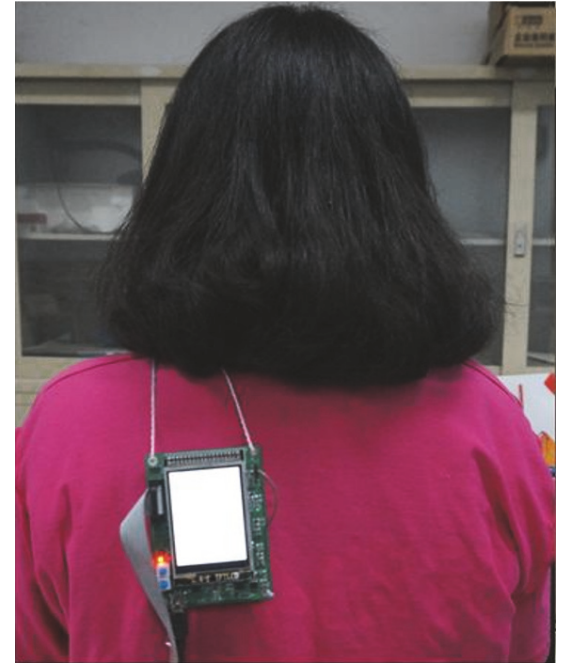

(a)

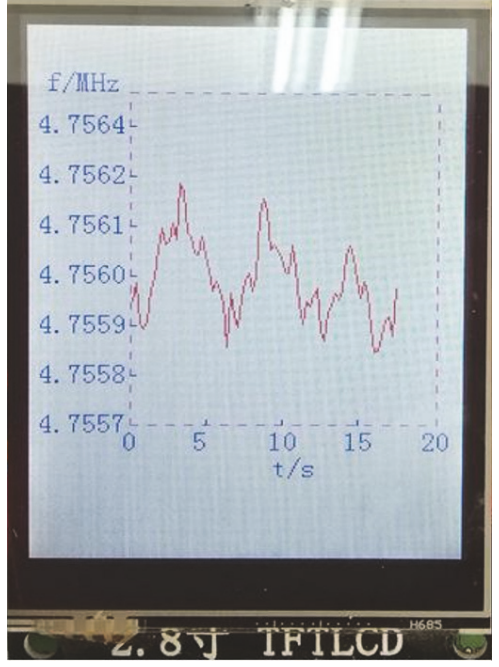

(b)

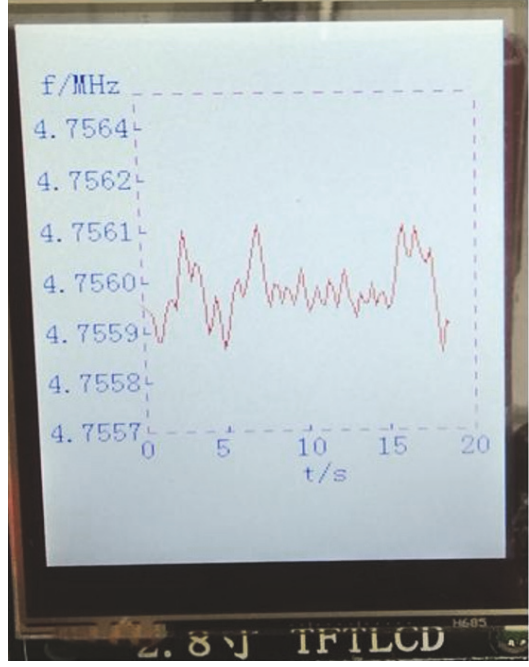

(c)

FiguRE 8: Cardiopulmonary signal waveform in different states: (a) the subject with the board, (b) prone position, normal breathing, and deep breathing, and (c) sitting, normal breathing, and apnea.

silently. He normally breathed $7 \mathrm{~s}$, held breath for about $7 \mathrm{~s}$, and recovered the normal cardiorespiratory status. The waveforms displayed that the frequency change in normal breath was $180 \mathrm{~Hz} 220 \mathrm{~Hz}$ and the frequency change in holding breath is $48 \mathrm{~Hz} \sim 60 \mathrm{~Hz}$, shown in Figure 8(c).

In the normal breathing, the changes in thoracic conductivity are greater than the changes in electrical conductivity caused by heart motion. Therefore, the frequency change in normal breath is mainly due to the respiratory activity, and the frequency change in holding breath is caused by heart motion. Compared with the experiment results in Figure 6, frequency change at normal breath is about $280 \sim 360 \mathrm{~Hz}$, and frequency variation due to holding breath is about $45 \sim 75 \mathrm{~Hz}$. According to our system measuring rules, the amplitude change corresponding to the frequency change is about $5.09 \mathrm{mV} \sim 6.21 \mathrm{mV}$ in normal breathing, $7.91 \mathrm{mV} \sim 10.18 \mathrm{mV}$ at deep inspiration, and $1.27 \mathrm{mV} \sim 2.09 \mathrm{mV}$ when the person is holding breath (Figure 8).

4.3.2. Cardiopulmonary Signal Estimation. Another experiment was conducted for evaluating the system performance. We compared frequency variation related to heart and lung activity detected by our system with the other representing cardiopulmonary state physiology signal BCG, which also reflects the respiratory behavior variation [17]. We obtained the BCG data and frequency variation due to electromagnetic induction by our designed system when the target object is at normal breath state and holding breath state.

Figures 9 and 10 show 10 -second recordings of the BCG signal and frequency change variation due to thoracic volume.

In Figure 9(a), the blue line is BCG signal, which can be seen as the ground truth, and red dashed line represented the respiratory waveform envelope. And in Figure 9(b), the blue line is frequency change due to cardiopulmonary activities by

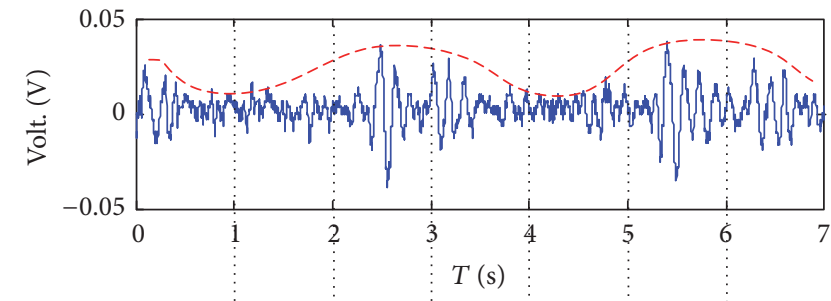

(a)

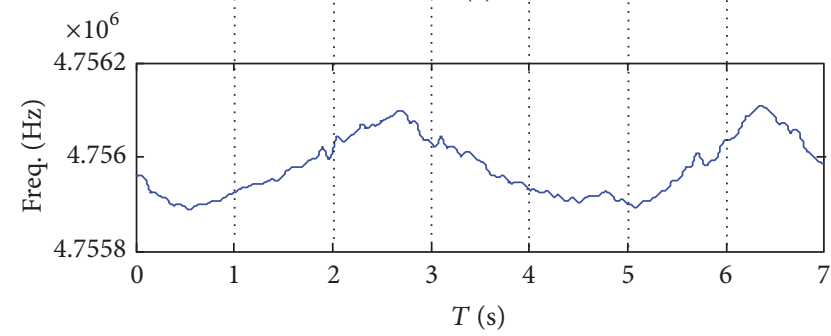

(b)

FIGURE 9: Normal respiratory signal: (a) BCG signal and (b) system measurement signal.

the designed system in this paper. Figure 10 shows the frequency variation and BCG signal when the object is holding breath. Obviously, the frequency change represents the heart and lung activity at two states.

In order to determine whether to use the frequency change detected by magnetic induction for estimation of respiratory rate and breath rate, we also analyze the signal frequency by FFT. Welch periodogram of the 15-second recording signal was shown in Figures 9 and 10. Normally, the respiratory frequency is about $12 \sim 20 \mathrm{bmp}$, and heart rate is about 60-100 bmp for normal adults. In Figure 11, there are two large peaks at $0.297 \mathrm{~Hz}$ and $1.12 \mathrm{~Hz}$. The corresponding 


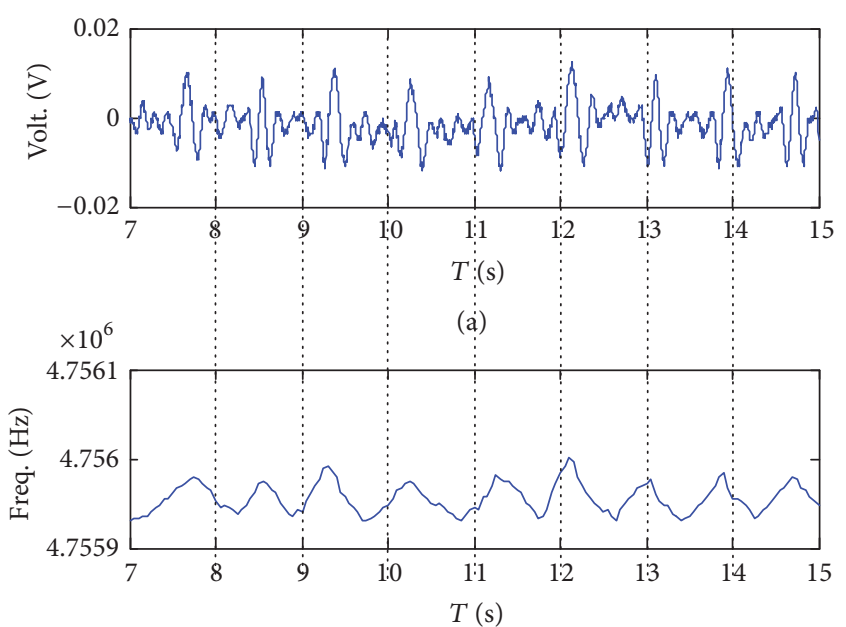

(b)

FIGURE 10: Holding breath signal: (a) BCG signal and (b) system measurement signal.

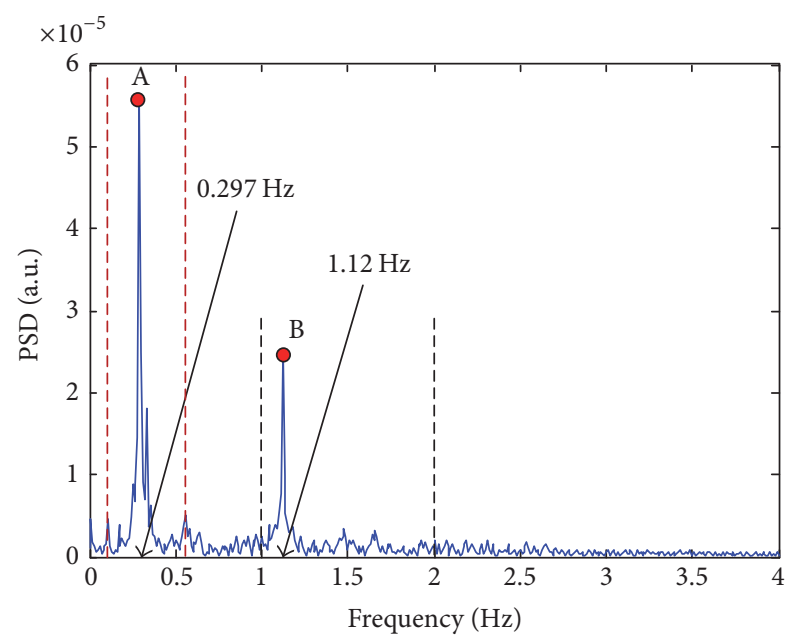

FIGURE 11: Welch period-gram (respiratory rate $=0.297 \mathrm{~Hz}$ and heart rate $=1.12 \mathrm{~Hz}$ ).

respiratory and heart rate are $18 \mathrm{bmp}$ and $67 \mathrm{bmp}$. According to Figure 10, in the 8-second holding breath, the waveform changes 9 times due to heart rate. The heart rate of the object is $67 \mathrm{bmp}$. The results obtained by frequency analysis are basically the same.

\section{Conclusion}

In this paper, we studied the problem of cardiopulmonary signal detection human localization by electromagnetic induction. We proposed a method that uses oscillator circuits to couple impedance change information due to breathing from coil sensors for human cardiopulmonary signal detection. The human impedance change due to thoracic volume variation can be coupled by coil sensors. Then oscillator frequency changed with the equivalent impedance of the couple coil. Our method has the advantage of reducing the obtrusiveness to the target object, while maintaining for detecting signals. We conducted experiments in a hardware platform and the feasibility and validity of the proposed method are evaluated. In the future, we will further improve our testbed and signal analysis method. For example, we need to minimize the board, and also we will also investigate the algorithm suitable for lying in a smart home environment.

\section{Conflicts of Interest}

The authors declare that they have no conflicts of interest.

\section{Acknowledgments}

The authors thank the National Natural Science Foundation of China (Grant no. 51607029) for supporting this research and the Fundamental Research Funds for the Central Universities of China under Grant nos. N150404003 and N160404009.

\section{References}

[1] World Health Organization, 10 Facts on Ageing and the Life Course, 2014, http://www.who.int/features/factfiles/ageing/ ageing_facts/en/.

[2] United Nations Population Fund, Ageing in the Twenty-First Century: A Celebration and a Challenge, 2012.

[3] G. Zhang, T. Wu, Z. Wan et al., "A method to differentiate between ventricular fibrillation and asystole during chest compressions using artifact-corrupted ECG alone," Computer Methods and Programs in Biomedicine, vol. 141, pp. 111-117, 2017.

[4] J. A. Stockley, A. M. Ismail, S. M. Hughes, R. Edgar, R. A. Stockley, and E. Sapey, "Maximal mid-expiratory flow detects early lung disease in $\alpha_{1}$-antitrypsin deficiency," Europen Respiratory Journal, vol. 49, no. 3, Article ID 1602055, 2017.

[5] Y. Nam, Y. Kong, B. Reyes, N. Reljin, and K. H. Chon, "Monitoring of heart and breathing rates using dual cameras on a smartphone," PLoS ONE, vol. 11, no. 3, Article ID e0151013, 2016, "Video-based respiration monitoring with automatic region of interest detection", Physiological Measurement.

[6] L. J. Goldman, "Nasal airflow and thoracoabdominal motion in children using infrared thermographic video processing," Pediatric Pulmonology, vol. 47, no. 5, pp. 476-486, 2012.

[7] S. L. Bennett, R. Goubran, and F. Knoefel, "The detection of breathing behavior using eulerian-enhanced thermal video," in Proceedings of the 37th Annual International Conference of the IEEE Engineering in Medicine and Biology Society, EMBC 2015, pp. 7474-7477, IEEE, Milan, Italy, 25-29 August 2015.

[8] K. Naishadham, J. E. Piou, L. Ren, and A. E. Fathy, "Estimation of cardiopulmonary parameters from ultra wideband radar measurements using the state space method," IEEE Transactions on Biomedical Circuits and Systems, vol. 10, no. 6, pp. 1037-1046, 2016.

[9] M. Steffen and S. Leonhardt, "Non-contact monitoring of heart and lung activity by magnetic induction measurement," Acta Polytechnica, vol. 48, no. 3, pp. 71-78, 2008.

[10] A. Cordes, M. Arts, and S. Leonhardt, "A full digital magnetic induction measurement device for non-contact vital parameter monitoring (MONTOS)," in Proceedings of the 34th Annual International Conference of the IEEE Engineering in Medicine 
and Biology Society, EMBS 2012, pp. 582-585, IEEE, San Diego, CA, USA, 28 August-1 September 2012.

[11] A. Cordes, M. Arts, and S. Leonhardt, "A neonatal thorax phantom for contact-less magnetic induction vitalparameter monitoring," in Proceedings of the 34th Annual International Conference of the IEEE Engineering in Medicine and Biology Society, EMBS 2012, pp. 1161-1164, IEEE, San Diego, CA, USA, 28 August-1 September 2012.

[12] A. Cordes, K. Heimann, and S. Leonhardt, "Magnetic induction measurements with a six channel coil array for vital parameter monitoring," in Proceedings of the 34th Annual International Conference of the IEEE Engineering in Medicine and Biology Society, EMBS 2012, pp. 602-604, San Diego, CA, USA, 28 August-1 September 2012.

[13] H. Mahdavi and J. Rosell-Ferrer, "Magnetic induction-based sensor for vital sign detection," in Proceedings of the 13th Mediterranean Conference on Medical and Biological Engineering and Computing 2013, MEDICON 2013, vol. 41 of IFMBE Proceedings, pp. 1507-1510, Springer, September 2013.

[14] H. Mahdavi and J. Rosell-Ferrer, "A magnetic induction measurement system for adult vital sign monitoring: Evaluation of capacitive and inductive effects," Journal of Physics: Conference Series, vol. 434, Article ID 012085, 2013.

[15] D. Teichmann, D. De Matteis, T. Bartelt, M. Walter, and S. Leonhardt, "A bendable and wearable cardiorespiratory monitoring device fusing two noncontact sensor principles," IEEE Journal of Biomedical and Health Informatics, vol. 19, no. 3, pp. 784-793, 2015.

[16] D. Teichmann, J. Foussier, J. Jia, S. Leonhardt, and M. Walter, "Noncontact monitoring of cardiorespiratory activity by electromagnetic coupling," IEEE Transactions on Biomedical Engineering, vol. 60, no. 8, pp. 2142-2152, 2013.

[17] P. S. Luna and R. Pallas, "Automatic Concealed Heart Rate Detction from the BCG in Seated Position," IEEE Latin America Transactions, vol. 13, no. 3, pp. 583-588, 2015. 


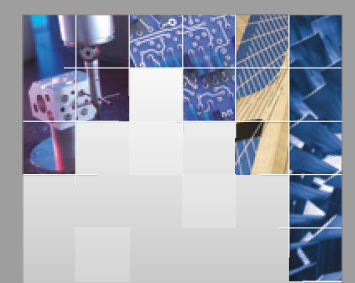

\section{Enfincering}
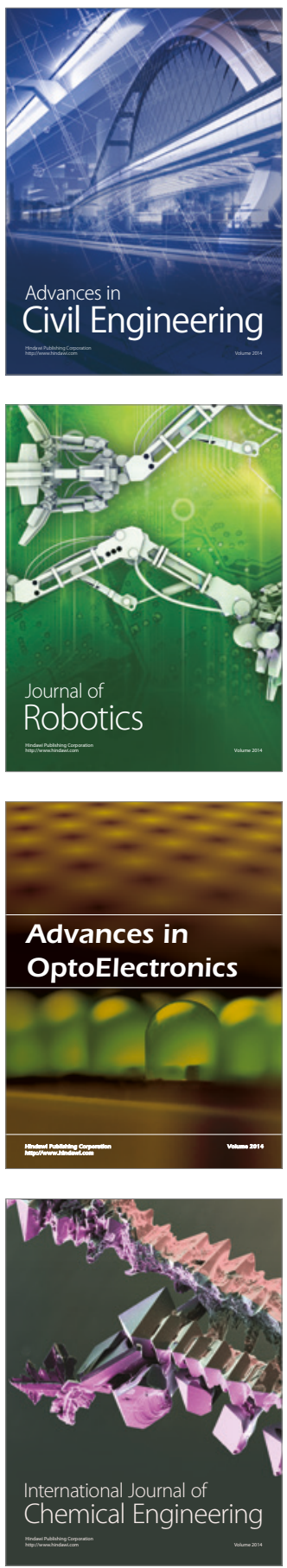

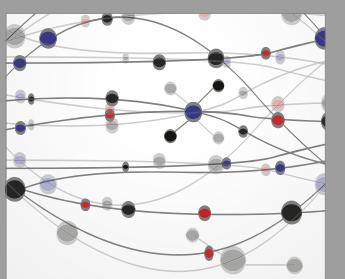

The Scientific World Journal

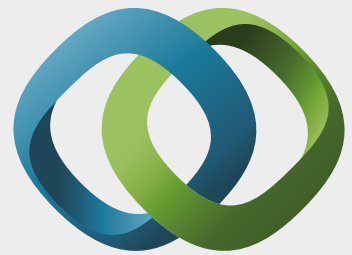

\section{Hindawi}

Submit your manuscripts at

https://www.hindawi.com
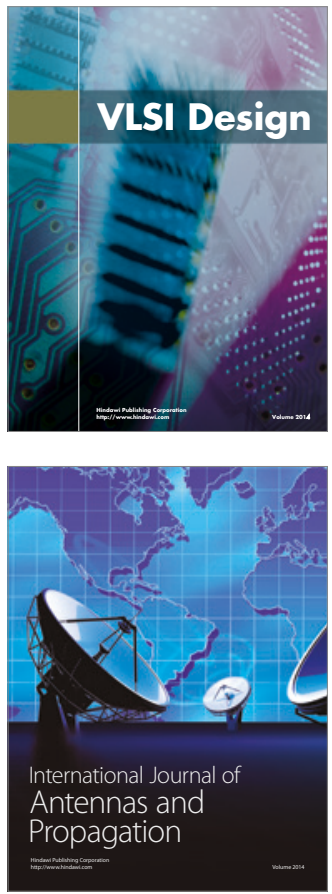

\section{Rotating}

Machinery
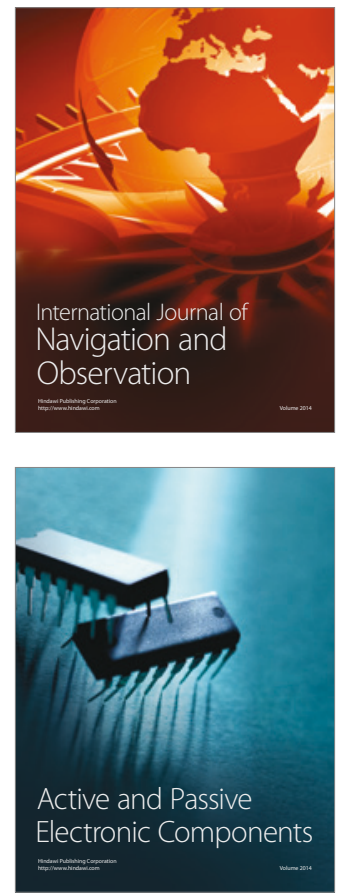
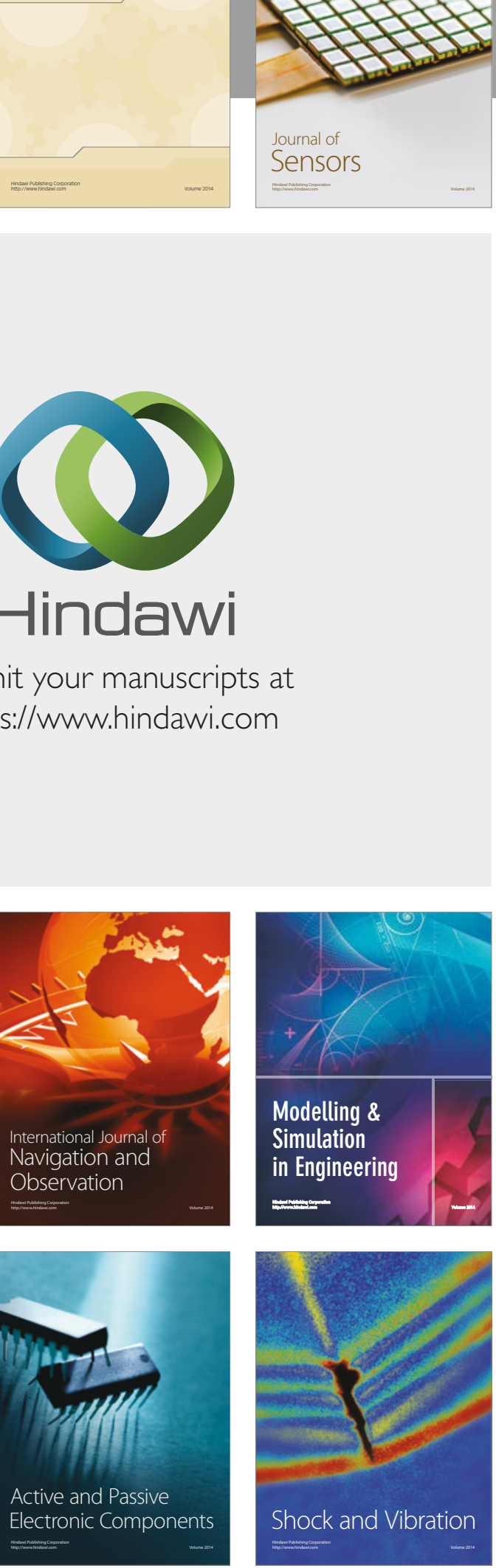
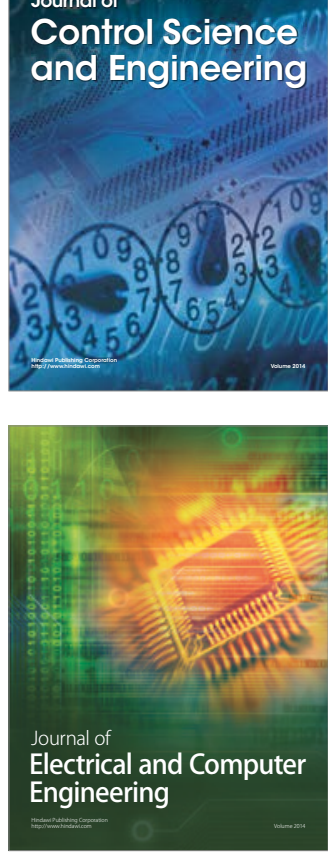

Distributed

Journal of

Control Science

and Engineering
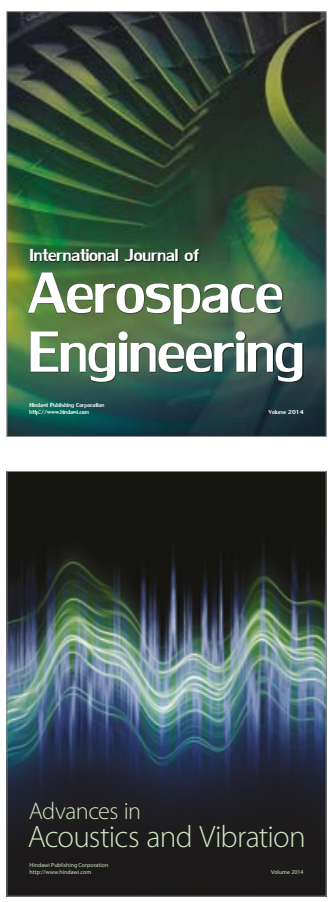

Sensor Networks 